\title{
Informational Divergence Approximations to Product Distributions
}

\author{
Jie Hou and Gerhard Kramer \\ Institute for Communications Engineering \\ Technische Universität München, 80290 Munich, Germany \\ Email: \{jie.hou, gerhard.kramer\}@tum.de
}

\begin{abstract}
The minimum rate needed to accurately approximate a product distribution based on an unnormalized informational divergence is shown to be a mutual information. This result subsumes results of Wyner on common information and Han-Verdú on resolvability. The result also extends to cases where the source distribution is unknown but the entropy is known.
\end{abstract}

\section{INTRODUCTION}

What is the minimal rate needed to generate a good approximation of a target distribution with respect to some distance measure? For example, to learn a system response, we might give inputs to the system and compute the output statistics. However, in computer simulations the inputs are only some approximations of the true distributions that are generated with random number generators. We would like to use a small number of bits to generate good approximations of a target distribution.

Wyner considered such a problem and characterized the smallest rate needed to approximate a product distribution accurately when using the normalized informational divergence as the distance measure between two distributions. The smallest rate is a Shannon mutual information [1]. Han-Verdú [2] showed that the same rate is necessary and sufficient to generate distributions arbitrarily close to an information stable distribution in terms of variational distance. Note that normalized informational divergence and variational distance are not necessarily larger or smaller than the other.

The main contributions of this work are to show that the minimal rate needed to make the unnormalized informational divergence between a target product distribution and the approximating distribution arbitrarily small is the same Shannon mutual information as in [1], [2] and we extend the proof to cases where the encoder has a non-uniform input distribution. Our result implies results in [1] and [2] when restricting attention to product distributions (in particular Theorem 6.3 in [1] and Theorem 4 in [2]). We remark that Hayashi developed closely related theory via Gallager's error exponent in [3] and Bloch and Kliewer considered non-uniform distributions for secrecy in [4]. We also refer to results by Csiszar [5, p. 44, bottom] who treats strong secrecy by showing that a variational distance exhibits an exponential behavior with block length $n$ [5, Prop. 2]. This result implies that an unnormalized mutual information expression can be made small with growing $n$ via [5] Lemma 1].

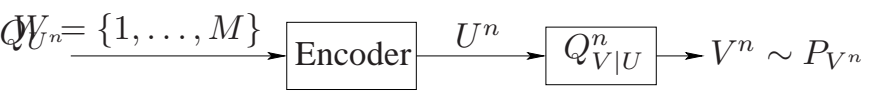

Fig. 1. Coding problem with the goal of making $P_{V^{n}} \approx Q_{V}^{n}$.

The paper is organized as follows. In Section II we state the problem. In Section III we state and prove the main result. Section IV] discusses related work and extensions.

\section{PRELIMINARIES}

Random variables are written with upper case letters and their realizations with the corresponding lower case letters. Superscripts denote finite-length sequences of variables/symbols, e.g., $X^{n}=X_{1}, \ldots, X_{n}$. Subscripts denote the position of a variable/symbol in a sequence. For instance, $X_{i}$ denotes the $i$-th variable in $X^{n}$. A random variable $X$ has probability distribution $P_{X}$ and the support of $P_{X}$ is denoted as $\operatorname{supp}\left(P_{X}\right)$. We write probabilities with subscripts $P_{X}(x)$ but we drop the subscripts if the arguments of the distribution are lower case versions of the random variables. For example, we write $P(x)=P_{X}(x)$. If the $X_{i}, i=1, \ldots, n$, are independent and identically distributed (i.i.d.) according to $P_{X}$, then we have $P\left(x^{n}\right)=\prod_{i=1}^{n} P_{X}\left(x_{i}\right)$ and we write $P_{X^{n}}=P_{X}^{n}$. Calligraphic letters denote sets. The size of a set $\mathcal{S}$ is denoted as $|\mathcal{S}|$. We use $\mathcal{T}_{\epsilon}^{n}\left(P_{X}\right)$ to denote the set of letter-typical sequences of length $n$ with respect to the probability distribution $P_{X}$ and the non-negative number $\epsilon$ [6, Ch. 3], [7], i.e., we have

$\mathcal{T}_{\epsilon}^{n}\left(P_{X}\right)=\left\{x^{n}:\left|\frac{N\left(a \mid x^{n}\right)}{n}-P_{X}(a)\right| \leq \epsilon P_{X}(a), \forall a \in \mathcal{X}\right\}$

where $N\left(a \mid x^{n}\right)$ is the number of occurrences of $a$ in $x^{n}$.

Consider the system depicted in Fig. 1. The random variable $W$ is uniformly distributed over $\{1, \ldots, M\}, M=2^{n R}$, and is encoded to sequences

$$
U^{n}=f(W) .
$$

$V^{n}$ is generated from $U^{n}$ through a memoryless channel $Q_{V \mid U}^{n}$ and has distribution $P_{V^{n}}$. A rate $R$ is achievable if for any $\xi>0$ there is a sufficiently large $n$ and an encoder such that

$$
D\left(P_{V^{n}} \| Q_{V}^{n}\right)=\sum_{v^{n} \in \operatorname{supp}\left(P_{V^{n}}\right)} P\left(v^{n}\right) \log \frac{P\left(v^{n}\right)}{Q_{V}^{n}\left(v^{n}\right)}
$$

is less than $\xi$. We wish to determine the smallest achievable rate. 


\section{Main Result and Proof}

Theorem 1: For a given target distribution $Q_{V}$, the rate $R$ is achievable if $R>I(V ; U)$, where $I(V ; U)$ is calculated with some joint distribution $Q_{U V}$ that has marginal $Q_{V}$ and $\left|\operatorname{supp}\left(Q_{U}\right)\right| \leq|\mathcal{V}|$. The rate $R$ is not achievable if $R<I(V ; U)$ for all $Q_{U V}$ with $\left|\operatorname{supp}\left(Q_{U}\right)\right| \leq|\mathcal{V}|$.

We provide two proofs, one with Shannon's typicality argument and the other with Gallager's error exponent [8] where we extend results in [3]. Suppose $U$ and $V$ have finite alphabets $\mathcal{U}$ and $\mathcal{V}$, respectively. Let $Q_{U V}$ be a probability distribution with marginals $Q_{U}$ and $Q_{V}$. Let $U^{n} V^{n} \sim Q_{U V}^{n}$, i.e., for any $u^{n} \in \mathcal{U}^{n}, v^{n} \in \mathcal{V}^{n}$ we have

$$
\begin{aligned}
& Q\left(u^{n}, v^{n}\right)=\prod_{i=1}^{n} Q_{U V}\left(u_{i}, v_{i}\right)=Q_{U V}^{n}\left(u^{n}, v^{n}\right) \\
& Q\left(v^{n} \mid u^{n}\right)=\prod_{i=1}^{n} Q_{V \mid U}\left(v_{i} \mid u_{i}\right)=Q_{V \mid U}^{n}\left(v^{n} \mid u^{n}\right) .
\end{aligned}
$$

Let $\mathcal{C}=\left\{U^{n}(w)\right\}_{w=1}^{M}$, where the $U^{n}(w), w=1, \ldots, M$, are generated in an i.i.d. manner using $Q_{U}^{n} \cdot V^{n}$ is generated from $U^{n}(W)$ through the channel $Q_{V \mid U}^{n}$ (see Fig. (2). We have

$$
P\left(v^{n}\right)=\sum_{w=1}^{M} \frac{1}{M} \cdot Q_{V \mid U}^{n}\left(v^{n} \mid u^{n}(w)\right) .
$$

Note that if for a $v^{n}$ we have

$$
Q_{V}^{n}\left(v^{n}\right)=\sum_{u^{n} \in \operatorname{supp}\left(Q_{U}^{n}\right)} Q_{U}^{n}\left(u^{n}\right) Q_{V \mid U}^{n}\left(v^{n} \mid u^{n}\right)=0
$$

then we have

$$
Q_{V \mid U}^{n}\left(v^{n} \mid u^{n}\right)=0 \text {, for all } u^{n} \in \operatorname{supp}\left(Q_{U}^{n}\right) .
$$

This means $P\left(v^{n}\right)=0$ and $\operatorname{supp}\left(P_{V^{n}}\right) \subseteq \operatorname{supp}\left(Q_{V}^{n}\right)$ so that $D\left(P_{V^{n}} \| Q_{V}^{n}\right)<\infty$. We further have

$$
\mathrm{E}\left[\frac{Q_{V \mid U}^{n}\left(v^{n} \mid U^{n}\right)}{Q_{V}^{n}\left(v^{n}\right)}\right]=\sum_{u^{n}} Q_{U}^{n}\left(u^{n}\right) \cdot \frac{Q_{V \mid U}^{n}\left(v^{n} \mid u^{n}\right)}{Q_{V}^{n}\left(v^{n}\right)}=1 .
$$

\section{A. Shannon's Typicality}

The average informational divergence over $W, \mathcal{C}$ and $V^{n}$ is (recall that $\left.P(w)=\frac{1}{M}, w=1, \ldots, M\right)$ :

$$
\begin{aligned}
& \mathrm{E}\left[D\left(P_{V^{n}} \| Q_{V}^{n}\right)\right] \stackrel{(a)}{=} \mathrm{E}\left[\log \frac{\sum_{j=1}^{M} \frac{1}{M} \cdot Q_{V^{n} \mid U^{n}}\left(V^{n} \mid U^{n}(j)\right)}{Q_{V}^{n}\left(V^{n}\right)}\right] \\
& =\sum_{w=1}^{M} \frac{1}{M} \cdot \mathrm{E}\left[\log \frac{\sum_{j=1}^{M} Q_{V \mid U}^{n}\left(V^{n} \mid U^{n}(j)\right)}{M Q_{V}^{n}\left(V^{n}\right)} \mid W=w\right] \\
& \stackrel{(b)}{\leq} \sum_{w=1}^{M} \frac{1}{M} \cdot \mathrm{E}\left[\log \left(\frac{Q_{V \mid U}^{n}\left(V^{n} \mid U^{n}(w)\right)}{M Q_{V}^{n}\left(V^{n}\right)}+\frac{M-1}{M}\right) \mid W=w\right] \\
& \leq \sum_{w=1}^{M} \frac{1}{M} \cdot \mathrm{E}\left[\log \left(\frac{Q_{V \mid U}^{n}\left(V^{n} \mid U^{n}(w)\right)}{M Q_{V}^{n}\left(V^{n}\right)}+1\right) \mid W=w\right] \\
& \stackrel{(c)}{=} \mathrm{E}\left[\log \left(\frac{Q_{V \mid U}^{n}\left(V^{n} \mid U^{n}\right)}{M \cdot Q_{V}^{n}\left(V^{n}\right)}+1\right)\right]
\end{aligned}
$$

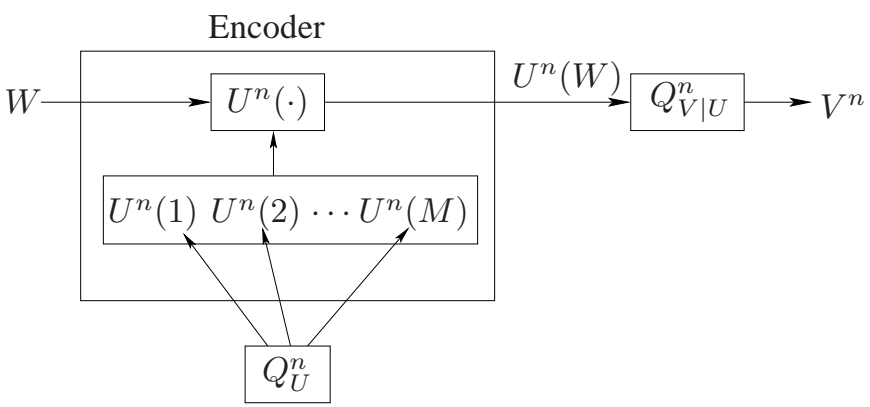

Fig. 2. The random coding experiment.

where

(a) follows by taking the expectation over $W, V^{n}$ and $U^{n}(1), \ldots, U^{n}(M)$;

(b) follows by the concavity of the logarithm and Jensen's inequality applied to the expectation over the $U^{n}(j), j \neq$ $w$, and by using (8);

(c) follows by choosing $U^{n} V^{n} \sim Q_{U V}^{n}$.

Alternatively, we can make the steps 9 more explicit:

$\mathrm{E}\left[D\left(P_{V^{n}} \| Q_{V}^{n}\right)\right] \stackrel{(a)}{=} \sum_{u^{n}(1)} \cdots \sum_{u^{n}(M)} \prod_{k=1}^{M} Q_{U}^{n}\left(u^{n}(k)\right)$

$\sum_{v^{n}} \sum_{w=1}^{M} \frac{1}{M} \cdot Q_{V \mid U}^{n}\left(v^{n} \mid u^{n}(w)\right)\left[\log \frac{\sum_{j=1}^{M} Q_{V \mid U}^{n}\left(v^{n} \mid u^{n}(j)\right)}{M \cdot Q_{V}^{n}\left(v^{n}\right)}\right]$

$=\sum_{w=1}^{M} \frac{1}{M} \sum_{v^{n}} \sum_{u^{n}(w)} Q_{U V}^{n}\left(u^{n}(w), v^{n}\right)$

$\sum_{k \neq w}^{M} \sum_{u^{n}(k)} \prod_{l \neq w}^{M} Q_{U}^{n}\left(u^{n}(l)\right)\left[\log \frac{\sum_{j=1}^{M} Q_{V \mid U}^{n}\left(v^{n} \mid u^{n}(j)\right)}{M \cdot Q_{V}^{n}\left(v^{n}\right)}\right]$

$\stackrel{(b)}{\leq} \sum_{w=1}^{M} \frac{1}{M} \sum_{v^{n}} \sum_{u^{n}(w)} Q_{U V}^{n}\left(u^{n}(w), v^{n}\right)$

$\left[\log \left(\frac{Q_{V \mid U}^{n}\left(v^{n} \mid u^{n}(w)\right)}{M \cdot Q_{V}^{n}\left(v^{n}\right)}+\sum_{j \neq w}^{M} \sum_{u^{n}(j)}\left[\frac{Q_{U V}^{n}\left(u^{n}(j), v^{n}\right)}{M \cdot Q_{V}^{n}\left(v^{n}\right)}\right]\right)\right]$

$=\sum_{w=1}^{M} \frac{1}{M} \sum_{v^{n}} \sum_{u^{n}(w)} Q_{U V}^{n}\left(u^{n}(w), v^{n}\right)$

$\left[\log \left(\frac{Q_{V \mid U}^{n}\left(v^{n} \mid u^{n}(w)\right)}{M \cdot Q_{V}^{n}\left(v^{n}\right)}+\frac{M-1}{M}\right)\right]$

$\leq \sum_{w=1}^{M} \frac{1}{M} \sum_{v^{n}} \sum_{u^{n}(w)} Q_{U V}^{n}\left(u^{n}(w), v^{n}\right)$

$\left[\log \left(\frac{Q_{V \mid U}^{n}\left(v^{n} \mid u^{n}(w)\right)}{M \cdot Q_{V}^{n}\left(v^{n}\right)}+1\right)\right]$

$\stackrel{(c)}{=} \mathrm{E}\left[\log \left(\frac{Q_{V \mid U}^{n}\left(V^{n} \mid U^{n}\right)}{M \cdot Q_{V}^{n}\left(V^{n}\right)}+1\right)\right]$.

We remark that the identity after $(a)$ is valid for $M=1$ by interpreting the empty sum followed by an empty product to 
be 1 . We may write 9 or 10 as

$$
\mathrm{E}\left[\log \left(\frac{Q_{V \mid U}^{n}\left(V^{n} \mid U^{n}\right)}{M \cdot Q_{V}^{n}\left(V^{n}\right)}+1\right)\right]=d_{1}+d_{2}
$$

where

$$
\begin{aligned}
& d_{1}= \sum_{\substack{\left(u^{n}, v^{n}\right) \in \mathcal{T}_{\epsilon}^{n}\left(Q_{U V}\right) \\
d_{2}=}} Q\left(u^{n}, v^{n}\right) \log \left(\frac{Q\left(v^{n} \mid u^{n}\right)}{M \cdot Q\left(v^{n}\right)}+1\right) \\
& \sum_{\substack{\left(u^{n}, v^{n}\right) \notin \mathcal{T}_{\epsilon}^{n}\left(Q_{U V}\right) \\
\left(u^{n}, v^{n}\right) \in \operatorname{supp}\left(Q_{U V}^{n}\right)}} Q\left(u^{n}, v^{n}\right) \log \left(\frac{Q\left(v^{n} \mid u^{n}\right)}{M \cdot Q\left(v^{n}\right)}+1\right) .
\end{aligned}
$$

Using standard inequalities (see [7]) we have

$$
\begin{aligned}
d_{1} & \leq \sum_{\left(u^{n}, v^{n}\right) \in \mathcal{T}_{\epsilon}^{n}\left(Q_{U V}\right)} Q\left(u^{n}, v^{n}\right) \log \left(\frac{2^{-n(1-\epsilon) H(V \mid U)}}{M \cdot 2^{-n(1+\epsilon) H(V)}}+1\right) \\
& \leq \log \left(\frac{2^{-n(1-\epsilon) H(V \mid U)}}{M \cdot 2^{-n(1+\epsilon) H(V)}}+1\right) \\
& =\log \left(2^{-n(R-I(V ; U)-\epsilon(H(V \mid U)+H(V)))}+1\right) \\
& \leq \log (e) \cdot 2^{-n(R-I(V ; U)-2 \epsilon H(V))}
\end{aligned}
$$

and $d_{1} \rightarrow 0$ if $R>I(V ; U)+2 \epsilon H(V)$ and $n \rightarrow \infty$. We further have

$$
\begin{aligned}
d_{2} & \sum_{\substack{\left(u^{n}, v^{n}\right) \notin \mathcal{T}_{\epsilon}^{n}\left(Q_{U V}\right) \\
\left(u^{n}, v^{n}\right) \in \operatorname{supp}\left(Q_{U V}^{n}\right)}} Q\left(u^{n}, v^{n}\right) \log \left(\left(\frac{1}{\mu_{V}}\right)^{n}+1\right) \\
& \leq 2|\mathcal{V}| \cdot|\mathcal{U}| \cdot e^{-2 n \epsilon^{2} \mu_{U V}^{2}} \log \left(\left(\frac{1}{\mu_{V}}\right)^{n}+1\right) \\
& \leq 2|\mathcal{V}| \cdot|\mathcal{U}| \cdot e^{-2 n \epsilon^{2} \mu_{U V}^{2}} \cdot n \cdot \log \left(\frac{1}{\mu_{V}}+1\right)
\end{aligned}
$$

and $d_{2} \rightarrow 0$ as $n \rightarrow \infty$, where

$$
\begin{aligned}
\mu_{V} & =\min _{v \in \operatorname{supp}\left(Q_{V}\right)} Q(v) \\
\mu_{U V} & =\min _{(v, u) \in \operatorname{supp}\left(Q_{U V}\right)} Q(u, v) .
\end{aligned}
$$

Combining the above we have

$$
\mathrm{E}\left[D\left(P_{V^{n}} \| Q_{V}^{n}\right)\right] \rightarrow 0
$$

if $R>I(V ; U)+2 \epsilon H(V)$ and $n \rightarrow \infty$. As usual, 17 means that there must exist a code with $D\left(P_{V^{n}} \| Q_{V}^{n}\right)<\xi$ for any $\xi>0$ and sufficiently large $n$. This proves the coding theorem. The converse follows from [1, Theorem 5.2] by removing the normalization factor $\frac{1}{n}$.

Remark 1: The cardinality bound on $\operatorname{supp}\left(Q_{U}\right)$. can be derived using techniques from [9, Ch. 15].

Remark 2: If $V=U$, then we have $R>H(V)$.

Theorem 1 is proved using a uniform $W$ which represents strings of uniform bits. If we use a non-uniform $W$ for the coding scheme, can we still drive the unnormalized informational divergence to zero? We give the answer in the following lemma.
Lemma 1: Let $W=B^{n R}$ be a bit stream with $n R$ bits that are generated i.i.d. with a binary distribution $P_{X}$ with $P_{X}(0)=p, 0<p \leq \frac{1}{2}$. The rate $R$ is achievable if

$$
R>\frac{I(V ; U)}{H_{2}(p)}
$$

where $H_{2}(\cdot)$ is the binary entropy function.

Proof: The proof is given in Appendix A

Remark 3: Lemma 1 states that even if $W$ is not uniformly distributed, the informational divergence can be made small. This is useful because if the distribution of $W$ is not known exactly, then we can choose $R$ large enough to guarantee the desired resolvability result. A similar result was developed in [4] for secrecy.

\section{B. Gallager's Error Exponent}

We provide a second proof using Gallager's error exponent [8] by extending [3, Lemma 2] to asymptotic cases. Consider $-\frac{1}{2} \leq \rho \leq 0$ and define

$$
\begin{aligned}
E_{0}^{n}\left(\rho, Q_{U V}^{n}\right) & =\log _{2} \sum_{v^{n}}\left\{\mathrm{E}\left[P\left(v^{n}\right)^{\frac{1}{1+\rho}}\right]\right\}^{1+\rho} \\
E_{0}\left(\rho, Q_{U V}\right) & =\log _{2} \sum_{v}\left\{\sum_{u} Q(u) Q(v \mid u)^{\frac{1}{1+\rho}}\right\}^{1+\rho} \\
E_{G}\left(R, Q_{U V}\right) & =\inf _{-\frac{1}{2} \leq \rho<0}\left\{E_{0}\left(\rho, Q_{U V}\right)+\rho R\right\} .
\end{aligned}
$$

Due to [3, Lemma 2], we have the following properties concerning $E_{0}^{n}\left(\rho, Q_{U V}^{n}\right)$ and $E_{0}\left(\rho, Q_{U V}\right)$ :

Property 1:

$$
E_{0}^{n}\left(0, Q_{U V}^{n}\right)=E_{0}\left(0, Q_{U V}\right)=0
$$

Property 2:

$$
\begin{aligned}
& \left.\frac{\partial E_{0}^{n}\left(\rho, Q_{U V}^{n}\right)}{\partial \rho}\right|_{\rho=0}=-\mathrm{E}\left[D\left(P_{V^{n}} \| Q_{V}^{n}\right)\right] \\
& \left.\frac{\partial E_{0}\left(\rho, Q_{U V}\right)}{\partial \rho}\right|_{\rho=0}=-I(V ; U)
\end{aligned}
$$

Property 3:

$$
\begin{aligned}
& \frac{\partial^{2} E_{0}^{n}\left(\rho, Q_{U V}^{n}\right)}{\partial \rho^{2}} \geq 0 \\
& \frac{\partial^{2} E_{0}\left(\rho, Q_{U V}\right)}{\partial \rho^{2}} \geq 0
\end{aligned}
$$

Due to [8, Theorem 5.6.3], we have

$$
\begin{cases}E_{G}\left(R, Q_{U V}\right)<0 & \text { if } R>I(V ; U) \\ E_{G}\left(R, Q_{U V}\right)=0 & \text { if } R \leq I(V ; U)\end{cases}
$$

By extending [3, Sec. III, Inequality (15)] to asymptotic cases, we have the following lemma.

Lemma 2: We have

$$
E_{0}^{n}\left(\rho, Q_{U V}^{n}\right) \leq \log _{2}\left(1+2^{n E_{G}\left(R, Q_{U V}\right)}\right) .
$$

Proof: The proof is given in Appendix B 


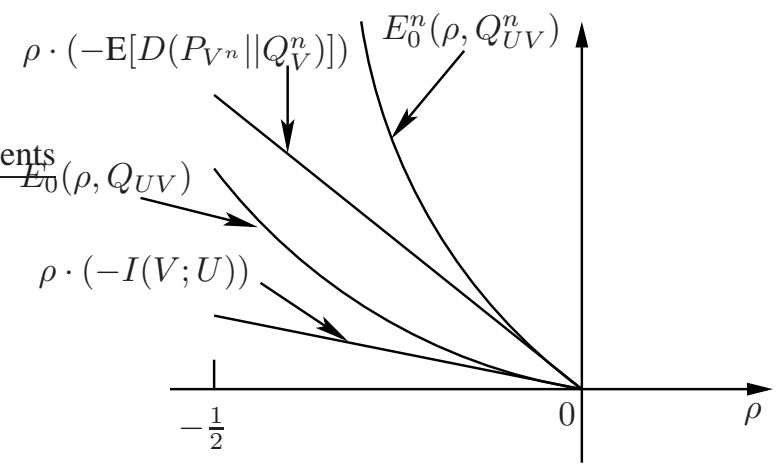

Fig. 3. An example of $E_{0}^{n}\left(\rho, Q_{U V}^{n}\right)$ and $E_{0}\left(\rho, Q_{U V}\right)$.

Combining Properties 1-3, we have $E_{0}^{n}\left(\rho, Q_{U V}^{n}\right)$ and $E_{0}\left(\rho, Q_{U V}\right)$ are convex in $\rho$, for $-\frac{1}{2} \leq \rho \leq 0$ and (see Fig. 3)

$$
\rho \cdot\left(-\mathrm{E}\left[D\left(P_{V^{n}} \| Q_{V}^{n}\right)\right]\right) \leq E_{0}^{n}\left(\rho, Q_{U V}^{n}\right)
$$

which means

$$
\begin{aligned}
\mathrm{E}\left[D\left(P_{V^{n}} \| Q_{V}^{n}\right)\right] & \leq \frac{E_{0}^{n}\left(\rho, Q_{U V}^{n}\right)}{-\rho} \\
& \stackrel{(a) \log _{2}\left(1+2^{n E_{G}\left(R, Q_{U V}\right)}\right)}{-\rho}
\end{aligned}
$$

where $(a)$ follows from Lemma2 The right hand side of 28 goes to 0 as $n \rightarrow \infty$ as long as (see 25)

$$
R>I(V ; U) .
$$

Remark 4: This proof applies to continuous random variables by replacing the sums in the proof of Lemma 2 with integrals.

Remark 5: The average divergence $\mathrm{E}\left[D\left(P_{V^{n}} \| Q_{V}^{n}\right)\right]$ can be viewed as the mutual information $I\left(\mathcal{C} ; V^{n}\right)$ from the random codebook $\mathcal{C}$ to the output $V^{n}$ [3, Sec. III]. To show this, denote $\widetilde{\mathcal{C}}$ as a realization of $\mathcal{C}$ and we have (see (10))

$$
\begin{aligned}
& I\left(\mathcal{C} ; V^{n}\right)=\sum_{\widetilde{\mathcal{C}}} P(\widetilde{\mathcal{C}}) \sum_{v^{n}} P\left(v^{n} \mid \widetilde{\mathcal{C}}\right) \log \frac{P\left(v^{n} \mid \widetilde{\mathcal{C}}\right)}{Q_{V}^{n}\left(v^{n}\right)} \\
& =\sum_{u^{n}(1)} \cdots \sum_{u^{n}(M)} \prod_{k=1}^{M} Q_{U}^{n}\left(u^{n}(k)\right) \\
& \sum_{v^{n}} \sum_{w=1}^{M} \frac{1}{M} \cdot Q_{V \mid U}^{n}\left(v^{n} \mid u^{n}(w)\right) \log \frac{\sum_{j=1}^{M} \frac{1}{M} Q_{V \mid U}^{n}\left(v^{n} \mid u^{n}(j)\right)}{Q_{V}^{n}\left(v^{n}\right)} \\
& =\mathrm{E}\left[\log \frac{\sum_{j=1}^{M} \frac{1}{M} Q_{V \mid U}^{n}\left(V^{n} \mid U^{n}(j)\right)}{Q_{V}^{n}\left(V^{n}\right)}\right] \\
& =\mathrm{E}\left[D\left(P_{V^{n}} \| Q_{V}^{n}\right)\right] .
\end{aligned}
$$

Thus, as $\mathrm{E}\left[D\left(P_{V^{n}} \| Q_{V}^{n}\right)\right] \rightarrow 0$ we have $I\left(\mathcal{C} ; V^{n}\right) \rightarrow 0$ which means that $\mathcal{C}$ and $V^{n}$ are (almost) independent. This makes sense, since as $P_{V^{n}} \rightarrow Q_{V}^{n}$ one is not able to distinguish which codebook is used to generate the output.

\section{DISCUSSION}

Hayashi studied the resolvability problem using unnormalized divergence and he derived bounds for nonasymptotic cases [3, Lemma 2]. We have outlined his proof steps in Sec. III-B Theorem 1 can be derived by extending [3, Lemma 2] to asymptotic cases (see $\amalg I-B$ ) and it seems that such a result was the underlying motivation for [3, Lemma 2]. Unfortunately, Theorem 1 is not stated explicitly in [3] and the ensuing asymptotic analysis was done for normalized informational divergence. Hayashi's proofs (he developed two approaches) were based on Shannon random coding.

Theorem 1 implies [1, Theorem 6.3] which states that for $R>I(V ; U)$ the normalized divergence $\frac{1}{n} D\left(P_{V^{n}} \| Q_{V}^{n}\right)$ can be made small. Theorem 1 implies [2, Theorem 4] for product distributions through Pinsker's inequality [10, Lemma 11.6.1]

$$
D\left(P_{X} \| Q_{X}\right) \geq \frac{1}{2 \ln 2}\left\|P_{X}-Q_{X}\right\|_{\mathrm{TV}}^{2}
$$

where

$$
\left\|P_{X}-Q_{X}\right\|_{\mathrm{TV}}=\sum_{x}|P(x)-Q(x)| .
$$

Moreover, the speed of decay in (12) and (14) is (almost) exponential with $n$. We can thus make

$$
\alpha(n) \cdot \mathrm{E}\left[D\left(P_{V^{n}} \| Q_{V}^{n}\right)\right]
$$

vanishingly small as $n \rightarrow \infty$, where $\alpha(n)$ represents a subexponential function of $n$ that satisfies,

$$
\lim _{n \rightarrow \infty} \frac{n \cdot \alpha(n)}{e^{\beta n}}=0
$$

where $\beta$ is positive and independent of $n$ (see also [3]). For example, we may choose $\alpha(n)=n^{m}$ for any integer $m$. We may also choose $\alpha(n)=e^{\gamma n}$ where $\gamma<\beta$.

Since all achievability results in [11] are based on [2, Theorem 4], Theorem 1 extends the results in [11] as well. Theorem 1 is closely related to strong secrecy [12] and provides a simple proof that Shannon random coding suffices to drive an unnormalized mutual information between messages and eavesdropper observations to zero.

Theorem 1 is valid for approximating product distributions only. However extensions to a broader class of distributions, e.g., information stable distributions [2], are clearly possible.

Finally, an example code is as follows (courtesy of F. Kschischang). Consider a channel with input and output alphabet the $2^{7}$ binary 7 -tuples. Suppose the channel maps each input uniformly to a 7 -tuple that is distance 0 or 1 away, i.e., there are 8 channel transitions for every input and each transition has probability $\frac{1}{8}$. A simple "modulation" code for this channel is the $(7,4)$ Hamming code. The code is perfect and if we choose each codeword with probability $\frac{1}{16}$, then the output $V^{7}$ of the channel is uniformly distributed over all $2^{7}$ values. Hence $I(V ; U)=4$ bits suffice to "approximate" the product distribution (here there is no approximation). 
APPENDIX A

\section{NON-UNIFORM $W$}

Observe that $H(W)=H\left(B^{n R}\right)=n R \cdot H_{2}(p)$. Following the same steps as in (9) we have

$$
\begin{aligned}
& \mathrm{E}\left[D\left(P_{V^{n}} \| Q_{V}^{n}\right)\right]=\mathrm{E}\left[\log \frac{\sum_{j=1}^{M} P(j) Q_{V^{n} \mid U^{n}}\left(V^{n} \mid U^{n}(j)\right)}{Q_{V}^{n}\left(V^{n}\right)}\right] \\
& =\sum_{w} P(w) \cdot \mathrm{E}\left[\log \frac{\sum_{j=1}^{M} P(j) Q_{V \mid U}^{n}\left(V^{n} \mid U^{n}(j)\right)}{Q_{V}^{n}\left(V^{n}\right)} \mid W=w\right] \\
& \leq \sum_{w} P(w) \cdot \mathrm{E}\left[\log \left(\frac{P(w) Q_{V \mid U}^{n}\left(V^{n} \mid U^{n}(w)\right)}{Q_{V}^{n}\left(V^{n}\right)}+1-P(w)\right)\right] \\
& \leq \sum_{w} P(w) \cdot \mathrm{E}\left[\log \left(\frac{P(w) Q_{V \mid U}^{n}\left(V^{n} \mid U^{n}(w)\right)}{Q_{V}^{n}\left(V^{n}\right)}+1\right)\right] \\
& =d_{1}+d_{2}+d_{3}
\end{aligned}
$$

where

$$
\begin{aligned}
& d_{1}=\sum_{w \in \mathcal{T}_{\epsilon}^{n}\left(P_{X}^{n}\right)} P(w) \sum_{\left(u^{n}(w), v^{n}\right) \in \mathcal{T}_{\epsilon}^{n}\left(Q_{U V}^{n}\right)} Q_{U V}^{n}\left(u^{n}(w), v^{n}\right) \\
& {\left[\log \left(\frac{P(w) Q_{V \mid U}^{n}\left(v^{n} \mid u^{n}(w)\right)}{Q_{V}^{n}\left(v^{n}\right)}+1\right)\right]} \\
& d_{2}=\sum_{w \in \mathcal{T}_{\epsilon}^{n}\left(P_{X}^{n}\right)} P(w) \sum_{\substack{\left(u^{n}(w), v^{n}\right) \notin \mathcal{T}_{\epsilon}^{n}\left(Q_{U V}\right) \\
\left(u^{n}(w), v^{n}\right) \in \operatorname{supp}\left(Q_{U V}^{n}\right)}} Q_{U V}^{n}\left(u^{n}(w), v^{n}\right) \\
& {\left[\log \left(\frac{P(w) Q_{V \mid U}^{n}\left(v^{n} \mid u^{n}(w)\right)}{Q_{V}^{n}\left(v^{n}\right)}+1\right)\right]} \\
& d_{3}=\sum_{w \notin \mathcal{T}_{\epsilon}^{n}\left(P_{X}^{n}\right)} P(w) \sum_{\left(u^{n}(w), v^{n}\right) \in \operatorname{supp}\left(Q_{U V}^{n}\right)} Q_{U V}^{n}\left(u^{n}(w), v^{n}\right) \\
& w \in \operatorname{supp}\left(P_{X}^{n}\right) \\
& {\left[\log \left(\frac{P(w) Q_{V \mid U}^{n}\left(v^{n} \mid u^{n}(w)\right)}{Q_{V}^{n}\left(v^{n}\right)}+1\right)\right] .}
\end{aligned}
$$

We can bound $d_{1}$ as follows (see (12) )

$$
\begin{aligned}
d_{1} & \leq \sum_{w \in \mathcal{T}_{\epsilon}^{n}\left(P_{X}^{n}\right)} P(w)\left[\log \left(\frac{2^{n(I(V ; U)+2 \epsilon H(V))}}{2^{n(1-\epsilon) R \cdot H_{2}(p)}}+1\right)\right] \\
& \leq \log \left(2^{-n\left(R \cdot H_{2}(p)-I(V ; U)-\epsilon\left(2 H(V)+R \cdot H_{2}(p)\right)\right)}+1\right) \\
& \leq \log (e) \cdot 2^{-n\left(R \cdot H_{2}(p)-I(V ; U)-\delta_{\epsilon}(n)\right)}
\end{aligned}
$$

which goes to zero if $R>\frac{I(V ; U)+\delta_{\epsilon}(n)}{H_{2}(p)}$ and $n \rightarrow \infty$, where $\delta_{\epsilon}(n)=\epsilon\left(2 H(V)+R \cdot H_{2}(p)\right)$. We also have

$$
\begin{aligned}
d_{2} \leq & \sum_{w \in \mathcal{T}_{\epsilon}^{n}\left(P_{X}^{n}\right)} P(w) \sum_{\substack{\left(u^{n}(w), v^{n}\right) \notin \mathcal{T}_{\epsilon}^{n}\left(Q_{U V}\right) \\
\left(u^{n}(w), v^{n}\right) \in \operatorname{supp}\left(Q_{U V}^{n}\right)}} Q_{U V}^{n}\left(u^{n}(w), v^{n}\right) \\
& {\left[\log \left(\left(\frac{1}{\mu_{V}}\right)^{n}+1\right)\right] } \\
\leq & 2|\mathcal{V}| \cdot|\mathcal{U}| \cdot e^{-2 n \epsilon^{2} \mu_{U V}^{2}} \log \left(\left(\frac{1}{\mu_{V}}\right)^{n}+1\right)
\end{aligned}
$$

which goes to zero as $n \rightarrow \infty$ (see (14)). We further have

$$
\begin{aligned}
d_{3} \leq & \sum_{\substack{w \notin \mathcal{T}_{\epsilon}^{n}\left(P_{X}^{n}\right) \\
w \in \operatorname{supp}\left(P_{X}^{n}\right)}} P(w) \sum_{\left(u^{n}(w), v^{n}\right) \in \operatorname{supp}\left(Q_{U V}^{n}\right)} Q_{U V}^{n}\left(u^{n}(w), v^{n}\right) \\
& {\left[\log \left(\left(\frac{1}{\mu_{V}}\right)^{n}+1\right)\right] } \\
\leq & \sum_{\substack{w \notin \mathcal{T}_{\epsilon}^{n}\left(P_{X}^{n}\right) \\
w \in \operatorname{supp}\left(P_{X}^{n}\right)}} P(w)\left[\log \left(\left(\frac{1}{\mu_{V}}\right)^{n}+1\right)\right] \\
\leq & 4 \cdot e^{-2 n \epsilon^{2} p^{2}} \log \left(\left(\frac{1}{\mu_{V}}\right)^{n}+1\right)
\end{aligned}
$$

which goes to zero as $n \rightarrow \infty$ (see (14)).

Combining the above for non-uniform $W$ we have

$$
\mathrm{E}\left[D\left(P_{V^{n}} \| Q_{V}^{n}\right)\right] \rightarrow 0
$$

if $R>\frac{I(V ; U)+\delta_{n}(\epsilon)}{H_{2}(p)}$ and $n \rightarrow \infty$.

\section{APPENDIX B}

\section{PROOF OF LEMMA 2}

We extend the proof of [3, Sec. III, Inequality (15)] to asymptotic cases to establish Lemma 2 . Recall that $-\frac{1}{2} \leq$ $\rho \leq 0$. Let $s=\frac{-\rho}{1+\rho}$ so we have

$$
\begin{aligned}
& 0 \leq s \leq 1 \\
& 1+s=\frac{1}{1+\rho}
\end{aligned}
$$

We also have for any $a, b \geq 0$ and $0 \leq x \leq 1$

$$
(a+b)^{x} \leq a^{x}+b^{x} .
$$

Observe that for any $v^{n}$ we have

$$
\begin{aligned}
\mathrm{E}\left[P\left(v^{n}\right)\right] & =\mathrm{E}\left[\sum_{w=1}^{M} \frac{1}{M} \cdot Q_{V \mid U}^{n}\left(v^{n} \mid U^{n}(w)\right)\right] \\
& =\mathrm{E}\left[Q_{V \mid U}^{n}\left(v^{n} \mid U^{n}(1)\right)\right] \\
& =\mathrm{E}\left[\prod_{i=1}^{n} Q_{V \mid U}\left(v_{i} \mid U_{i}(1)\right)\right] \\
& =\prod_{i=1}^{n} \mathrm{E}\left[Q_{V \mid U}\left(v_{i} \mid U_{i}(1)\right)\right] \\
& =\prod_{i=1}^{n}\left[\sum_{u} Q(u) Q_{V \mid U}\left(v_{i} \mid u\right)\right] \\
& =\prod_{i=1}^{n} Q_{V}\left(v_{i}\right)=Q_{V}^{n}\left(v^{n}\right)
\end{aligned}
$$


We further have

$$
\begin{aligned}
& 2^{E_{0}^{n}\left(\rho, Q_{U V}^{n}\right)}=\sum_{v^{n}}\left\{\mathrm{E}\left[P\left(v^{n}\right)^{\frac{1}{1+\rho}}\right]\right\}^{1+\rho} \\
& \stackrel{(a)}{=} \sum_{v^{n}}\left\{\mathrm{E}\left[P\left(v^{n}\right)^{1+s}\right]\right\}^{\frac{1}{1+s}} \\
& =\sum_{v^{n}}\left\{\mathrm{E}\left[\left(\sum_{w=1}^{M} \frac{1}{M} \cdot Q_{V \mid U}^{n}\left(v^{n} \mid U^{n}(w)\right)\right)^{1+s}\right]\right\}^{\frac{1}{1+s}} \\
& =\frac{1}{M} \sum_{v^{n}}\left\{\mathrm { E } \left[\sum_{w=1}^{M} Q_{V \mid U}^{n}\left(v^{n} \mid U^{n}(w)\right)\right.\right. \\
& \left.\left.\left(Q_{V \mid U}^{n}\left(v^{n} \mid U^{n}(w)\right)+\sum_{j \neq w}^{M} Q_{V \mid U}^{n}\left(v^{n} \mid U^{n}(j)\right)\right)\right]\right\}^{\frac{1}{1+s}}
\end{aligned}
$$

where $(a)$ follows from (41). Applying (42) to (44) we have

$$
\begin{aligned}
& 2^{E_{0}^{n}\left(\rho, Q_{U V}^{n}\right)} \leq \frac{1}{M} \sum_{v^{n}}\left\{\mathrm { E } \left[\sum_{w=1}^{M} Q_{V \mid U}^{n}\left(v^{n} \mid U^{n}(w)\right)\right.\right. \\
& \left.\left.\left(\left(Q_{V \mid U}^{n}\left(v^{n} \mid U^{n}(w)\right)\right)^{s}+\left(\sum_{j \neq w}^{M} Q_{V \mid U}^{n}\left(v^{n} \mid U^{n}(j)\right)\right)^{s}\right)\right]\right\}^{\frac{1}{1+s}} \\
& \stackrel{(a)}{=} \frac{1}{M} \sum_{v^{n}}\left\{\mathrm{E}\left[\sum_{w=1}^{M}\left(Q_{V \mid U}^{n}\left(v^{n} \mid U^{n}(w)\right)\right)^{1+s}\right]\right. \\
& +\sum_{w=1}^{M}\left(\mathrm{E}\left[Q_{V \mid U}^{n}\left(v^{n} \mid U^{n}(w)\right)\right]\right) \\
& \left.\cdot \mathrm{E}\left[\left(\sum_{j \neq w}^{M} Q_{V \mid U}^{n}\left(v^{n} \mid U^{n}(j)\right)\right)^{s}\right]\right\}^{\frac{1}{1+s}} \\
& \stackrel{(b)}{\leq} \frac{1}{M} \sum_{v^{n}}\left\{M \mathrm{E}\left[\left(Q_{V \mid U}^{n}\left(v^{n} \mid U^{n}\right)\right)^{1+s}\right]\right. \\
& \left.+M Q_{V}^{n}\left(v^{n}\right) \cdot\left(\mathrm{E}\left[\sum_{j \neq w}^{M} Q_{V \mid U}^{n}\left(v^{n} \mid U^{n}(j)\right)\right]\right)^{s}\right\}^{\frac{1}{1+s}} \\
& \stackrel{(c)}{=} \frac{1}{M} \sum_{v^{n}}\left\{M \mathrm{E}\left[\left(Q_{V \mid U}^{n}\left(v^{n} \mid U^{n}\right)\right)^{1+s}\right]\right. \\
& \left.+M Q_{V}^{n}\left(v^{n}\right)\left((M-1) Q_{V}^{n}\left(v^{n}\right)\right)^{s}\right\}^{\frac{1}{1+s}} \\
& \leq \frac{1}{M} \sum_{v^{n}}\left\{M \mathrm{E}\left[\left(Q_{V \mid U}^{n}\left(v^{n} \mid U^{n}\right)\right)^{1+s}\right]+\left(M Q_{V}^{n}\left(v^{n}\right)\right)^{1+s}\right\}^{\frac{1}{1+}}
\end{aligned}
$$

where

(a) follows because $U^{n}(w)$ is independent of $U^{n}(j), j \neq w$

(b) follows by choosing $U^{n} V^{n} \sim Q_{U V}^{n}$, by the concavity of $x^{a}$ for $0 \leq a \leq 1$ and by

(c) follows by 43
Applying (42) again to 45] we have

$$
\begin{aligned}
& 2^{E_{0}^{n}\left(\rho, Q_{U V}^{n}\right)} \leq \frac{1}{M} \sum_{v^{n}}\left\{\left(M \mathrm{E}\left[\left(Q_{V \mid U}^{n}\left(v^{n} \mid U^{n}\right)\right)^{1+s}\right]\right)^{\frac{1}{1+s}}\right. \\
& \left.+M Q_{V}^{n}\left(v^{n}\right)\right\} \\
& \stackrel{(a)}{=} 1+M^{\rho} \sum_{v^{n}}\left(\mathrm{E}\left[\left(Q_{V \mid U}^{n}\left(v^{n} \mid U^{n}\right)\right)^{\frac{1}{1+\rho}}\right]\right)^{1+\rho} \\
& =1+M^{\rho} \sum_{v^{n}}\left(\sum_{u^{n}} Q_{U}^{n}\left(u^{n}\right)\left(Q_{V \mid U}^{n}\left(v^{n} \mid u^{n}\right)\right)^{\frac{1}{1+\rho}}\right)^{1+\rho} \\
& \stackrel{(b)}{=} 1+2^{n \rho R} \sum_{v}\left(\sum_{u} Q(u)(Q(v \mid u))^{\frac{1}{1+\rho}}\right)^{n(1+\rho)} \\
& =1+2^{n\left(E_{0}\left(\rho, Q_{U V}\right)+\rho R\right)}
\end{aligned}
$$

where

(a) follows from 41

(b) follows because the $U_{i} V_{i}$ are i.i.d., $i=1, \ldots, n$

Optimizing over $\rho$, we have

$$
E_{0}^{n}\left(\rho, Q_{U V}^{n}\right) \leq \log _{2}\left(1+2^{n E_{G}\left(R, Q_{U V}\right)}\right) .
$$

\section{ACKNOWLEDGMENT}

J. Hou and G. Kramer were supported by an Alexander von Humboldt Professorship endowed by the German Federal Ministry of Education and Research. G. Kramer was also supported by NSF Grant CCF-09-05235. The authors thank G. Böcherer, F. Kschischang and M. Bloch for useful remarks.

\section{REFERENCES}

[1] A. Wyner, "The common information of two dependent random variables," IEEE Trans. Inf. Theory, vol. 21, no. 2, pp. 163-179, March 1975.

[2] T. Han and S. Verdú, "Approximation theory of output statistics," IEEE Trans. Inf. Theory, vol. 39, no. 3, pp. 752-772, May 1993.

[3] M. Hayashi, "General nonasymptotic and asymptotic formulas in channel resolvability and identification capacity and their application to the wiretap channel," IEEE Trans. Inf. Theory, vol. 52, no. 4, pp. 15621575, April 2006.

[4] M. Bloch and J. Kliewer, "On secure communication with constrained randomization," in IEEE Int. Symp. Inf. Theory, Boston, MA, USA, 2012, pp. 1172-1176.

[5] I. Csiszár, "Almost independence and secrecy capacity," Prob. of Inf. Transmission, vol. 32, no. 1, pp. 40-47, Jan.-March 1996.

[6] J. L. Massey, Applied Digital Information Theory, ETH Zurich, Zurich, Switzerland, 1980-1998.

[7] A. Orlitsky and J. Roche, "Coding for computing," IEEE Trans. Inf. Theory, vol. 47, no. 3, pp. 903-917, March 2001.

[8] R. G. Gallager, Information Theory and Reliable Communication. Wiley, 1968

[9] I. Csiszár and J. Körner, Information Theory: Coding Theorems for Discrete Memoryless Systems. New York: Academic, 1981.

[10] T. Cover and J. Thomas, Elements of Information Theory, 2nd ed. New York: Wiley, 2006

[11] P. Cuff, H. Permuter, and T. Cover, "Coordination capacity," IEEE Trans. Inf. Theory, vol. 56, no. 9, pp. 4181-4206, Sept. 2010.

[12] M. Bloch and J. Barros, Physical Layer Security From Information Theory to Security Engineering. Cambridge University Press, 2011. 